\title{
Multiple Low-Grade Fibromyxoid Sarcoma on the Upper Arms with Atypical Histological Presentation
}

\author{
Sadanori Furudate Taku Fujimura Yumi Kambayashi Akira Tsukada \\ Yukikazu Numata Setsuya Aiba \\ Department of Dermatology, Tohoku University Graduate School of Medicine, \\ Sendai, Japan
}

\section{Key Words}

Low-grade fibromyxoid sarcoma $\cdot$ Multiple recurrence $\cdot$ Palisaded granuloma-like bodies (rosettes)

\begin{abstract}
Low-grade fibromyxoid sarcoma (LGFMS) is a rare variant of spindle cell tumor that is composed of collagen-rich and myxoid parts. We describe the case of a 61-year-old Japanese patient with multiple, recurrent LGFMS on the upper arms with atypical histological presentation. In the present case, we resected the tumor several times with a minimal surgical margin, as in Moh's microsurgery. However, this can frequently lead to local recurrence of the tumor. Our case suggested that, regarding mesenchymal tumors with potential of malignancy in the skin, an initial wide excision is indispensable for complete remission of the tumor, even for low-grade malignancy such as LGFMS.
\end{abstract}

\section{Introduction}

Fibrosarcomas are mesenchymal neoplasms with fibrous differentiation that have a low-grade potential for malignancy. Various types of low-grade fibrosarcoma were previously reported: low-grade myxofibrosarcoma [1-3], low-grade fibromyxoid sarcoma (LGFMS), hyalinizing spindle cell tumor with giant rosettes [4, 5], sclerosing epithelioid fibrosarcoma, low-grade fibrosarcoma with palisaded granuloma-like bodies [6], and lowgrade fibrosarcomas not otherwise specified [7]. Among them, LGFMS is a rare variant of spindle cell tumor that is composed of collagen-rich and myxoid parts [1, 2]. LGFMS proliferates mainly in subcutaneous or superficial soft tissue on the proximal extremities in 
young to middle-aged adults. Clinically, the tumor sometimes recurs locally but rarely has a metastatic potential [6]. In this report, we present a case of multiple LGFMS on the upper arms with atypical histological presentation.

\section{Case Report}

A 61-year-old Japanese female presented to our clinic with an 8-month history of asymptomatic subcutaneous nodules on her arm. On her first visit to our outpatient clinic, physical examination revealed an elastic, hard subcutaneous nodule on her bilateral upper arms (fig. 1). The nodules were approximately $25 \mathrm{~mm}$ in diameter. We excised two nodules and histological findings revealed degenerated collagen fibers and necrosis, surrounded by histiocytes and partially palisaded granuloma-like bodies (collagen rosettes) (fig. 2a, b). We observed spindle-shaped cells that had eosinophilic cytoplasm, with no nuclear atypia or mitotic activity (fig. 2b). Mucin deposition was prominent at the centers of the granulomalike bodies (fig. 2c). We diagnosed this tumor as a subcutaneous type of granuloma annulare or myxofibroma. One year later, the patient noticed a subcutaneous nodule on her left arm and we excised the nodule again. Histological findings were similar to those of the previous lesion. A half year later, a subcutaneous nodule, $20 \mathrm{~mm}$ in diameter, appeared on her left arm near the operative area. Then, we screened for a possible internal malignancy with positron emission tomography but found none. We excised the nodule again below the muscle fascia, and the histopathological findings revealed myxoedematous proliferation, degenerated collagen fibers and necrosis, surrounded by lymphocytes, histiocytes and multinucleated giant cells in the dermal to subcutaneous area (fig. 2d). Alcian-blue PAS staining revealed mucin deposition at the center of the tumor. From the above data, we diagnosed this tumor as LGFMS. Two years after the last excision, there was no sign of recurrence or metastasis.

\section{Discussion}

In this report, we describe a case of multiple, recurrent low-grade fibromyxoid sarcoma on the upper arms. The histological findings of the nodules revealed degenerated collagen fibers and necrosis, surrounded by histiocytes and partially palisaded granuloma-like bodies (collagen rosettes), mimicking a subcutaneous type of generalized granuloma annulare. Unexpectedly, the nodules recurred several times at both local sites and sites remote from the resected area. Concerning the most recent nodule, histological findings revealed prominent mucin deposition at the center of the tumor. From the clinical course and histological findings, we finally diagnosed this patient with multiple, recurrent LGFMS on the arm.

LGFMS is a distinctive variant of fibrosarcoma that was first described by Evans [8]. It is characterized by spindle-shaped cells arranged in a whorled pattern with alternating fibrous and myxoid stroma. About 30\% of these tumors also develop collagen rosettes [9]. Local recurrence is seen in $9.3 \%$ of patients and metastasis occurs in $5.6 \%$ of patients [9]. In Japan, on the other hand, local recurrence is seen in $12.9 \%$ of patients and usually no metastasis occurs [10]. This discrepancy might be caused by differences in the types of LGFMS, since the concept of LGFMS has been developing during recent decades. For instance, Mansoor and White [11] reported another variant of subcutaneous malignancies similar to LMGFS, myxofibrosarcoma presenting in the skin, which is characterized by low-grade myxoid tumors with dermal involvement but no collagen rosettes. LGFMS and myxofibrosarcoma 
Furudate et al.: Multiple Low-Grade Fibromyxoid Sarcoma on the Upper Arms with Atypical Histological Presentation

might be misdiagnosed as benign dermal myxoid neoplasm, including superficial angiomyxoma, myxoid neurofibroma, nerve sheath myxoma, and myxoid dermatofibrosarcoma protuberans [12]. Interestingly, Folpe et al. [9] reported that in 15\% of patients with LGFMS, a histologic diagnosis was delayed by over 5 years, which suggested difficulties in diagnosing LGFMS. Our case was also confusing because from the beginning, the tumors were observed bilaterally on the upper arms, which strongly suggested an inflammatory disorder, such as a granulomatous disorder. After several excisional biopsies, these tumors revealed bilateral primary lesions of LGFMS. At the final excision, we excised the tumor below the muscle fascia, and there has been no sign of local or remote recurrence for 2 years. Our case suggested that for mesenchymal tumors with potential of malignancy in the skin, initial wide excision is indispensable for complete remission of the tumor, even for low-grade malignancy such as LGFMS.

\section{Acknowledgements}

The authors thank Prof. Martin C. Mihm Jr. (Pathology and Dermatology Director of Melanoma, Dermatology Department Brigham and Women's Hospital) for advice concerning the diagnosis of this case.

\section{Disclosure Statement}

There are no conflicts of interest.

\section{References}

$\checkmark 1$ Mentzel T, Calonje E, et al: Myxofibrosarcoma: clinicopathologic analysis of 75 cases with emphasis on the low-grade variant. Am J Surg Pathol 1996;20:391-405.

2 Oda Y, Takahira T, et al: Low-grade fibromyxoid sarcoma versus low-grade myxofibrosarcoma in the extremities and trunk. A comparison of clinicopathological and immunohistochemical features. Histopathology 2004;45:29-38.

-3 Angervall L, Kindblom LG, et al: Myxofibrosarcoma a study of 30 cases. Acta Pathol Microbiol Scand A 1977;85A:127-140.

4 Ramaswamy AS, Chatura KR: Hyalinizing spindle cell tumor with giant rosettes. Ann Saudi Med 2011;31:8386.

5 Franchi A, Massi D, Santucci M: Hyalinizing spindle cell tumor with giant rosettes and low-grade fibromyxoid sarcoma: an immunohistochemical and ultrastructual comperative investigation. Ultrastruct Pathol 2003;27:349-355.

-6 Woodruff JM, Antonescu CR: Low-grade fibrosarcoma with palisaded granuloma like bodies (giant rosettes). Report of a case that metastasized. Am J Surg Pathol 1999;23:1423-1428.

7 Hansen T, Katenkamp K, et al: Low-grade fibrosarcoma - report on 39 not otherwise specified cases and comparison with defined low-grade fibrosarcoma types. Histopathology 2006;49:152-160.

-8 Evans HL: Low-grade fibromyxoid sarcoma. A report of two metastasizing neoplasms having a deceptively benign appearance. Am J Clin Pathol 1987;88:615-619.

-9 Folpe AL, Lane KL, Paull G, Weis SW: Low-grade fibromyxoid sarcoma and hyalinizing spindle cell tumor with giant rosettes: a clinicopathologic study of 73 cases supporting their identity and assessing the impact of high-grade areas. Am J Surg Pathol 2000;24:1353-1360.

10 Suzumura T, Kato M, et al: A case of myxofibrosarcoma. Skin Cancer (Jpn) 2012;27:162-165.

11 Mansoor A, White CR Jr: Myxofibrosarcoma presenting in the skin: clinicopathological feature and differential diagnosis with cutaneous myxoid neoplasm. Am J Dermatopathol 2003;25:281.

12 Fujimura T, Okuyama R, Terui T, Okuno K, Masu A, Masu T, Chiba S, Kunii T, Tagami H, Aiba S: Myxofibrosarcoma (myxoid malignant fibrous histiocytoma) showing cutaneous presentation: report of two cases. J Cutan Pathol 2005;32:512-515. 


\section{Case Reports in Dermatology}

\begin{tabular}{l|l}
\hline \multicolumn{2}{l}{ Case Rep Dermatol 2013;5:152-155 } \\
\hline DOI: 10.1159/000351791 & $\begin{array}{l}\text { C 2013 S. Karger AG, Basel } \\
\text { www.karger.com/cde }\end{array}$ \\
\hline
\end{tabular}

Furudate et al.: Multiple Low-Grade Fibromyxoid Sarcoma on the Upper Arms with Atypical Histological Presentation

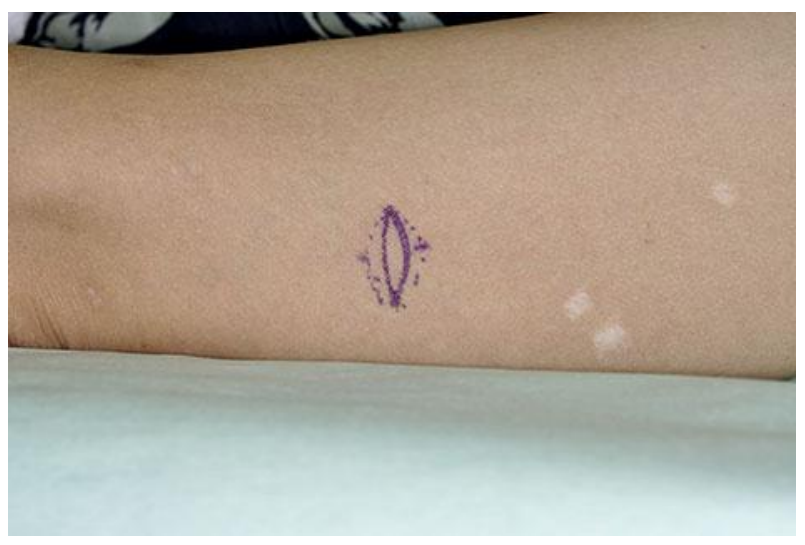

Fig. 1. An elastic, hard subcutaneous nodule on the bilateral upper arms is shown.
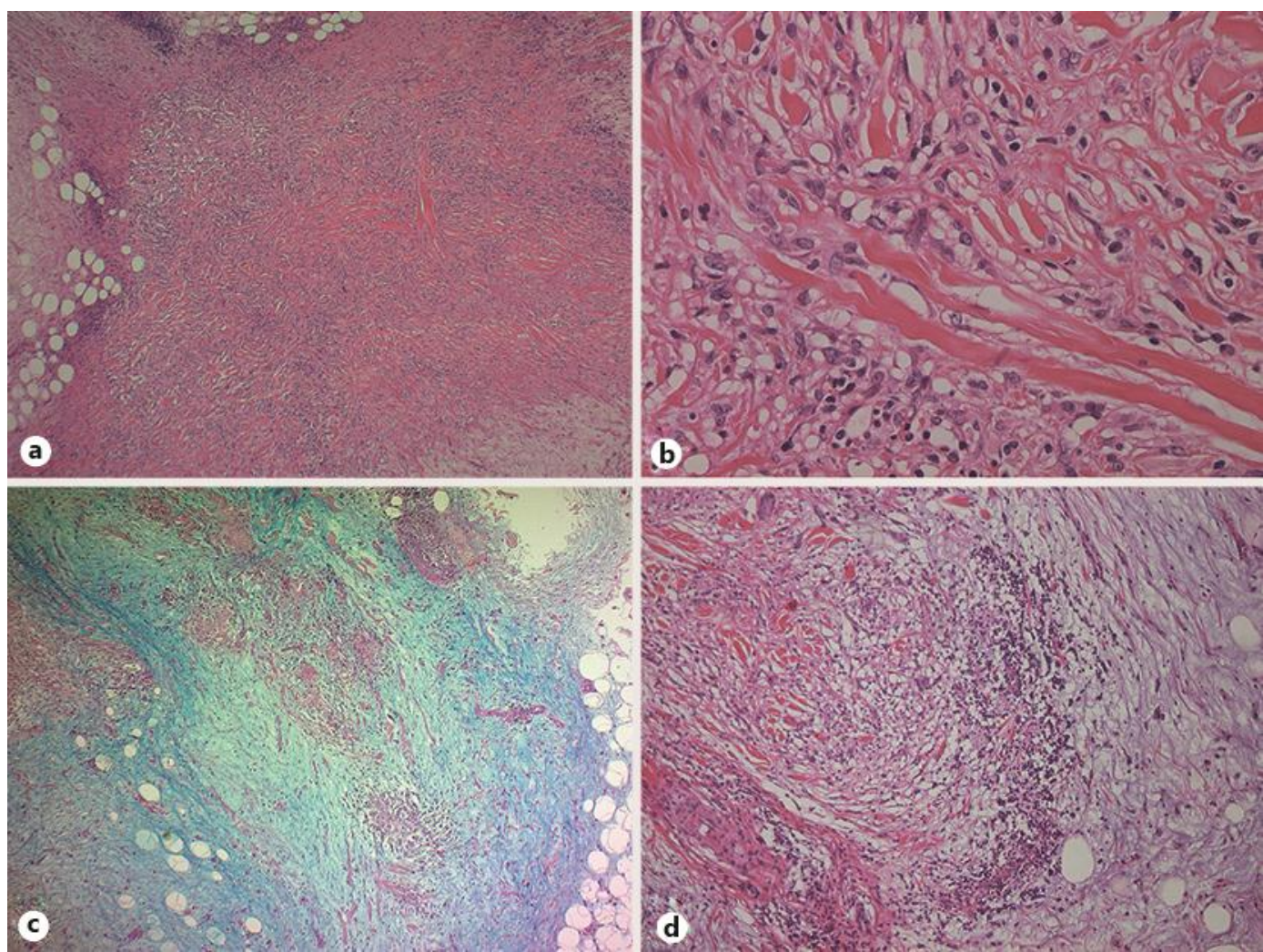

Fig. 2. Degenerated collagen fibers and necrosis surrounded by histiocytes and partially palisaded granuloma-like bodies (rosettes, a). Spindle-shaped cells with eosinophilic cytoplasm, showing no nuclear atypia or mitotic activity (b). Mucin deposition is prominent at the centers of the granuloma-like bodies (c). Myxoedematous proliferation, degenerated collagen fibers and necrosis surrounded by lymphocytes, histiocytes and multinucleated giant cells in the dermal to subcutaneous area, partially resembling a palisading granuloma (d). 